\title{
Spinal anesthesia and postoperative epidural analgesia in a patient with congenital central hypoventilation syndrome -a case report-
}

\author{
Yongjoon Choi, Sunam Lee, Jiyeon Lee, and Seongwon Woo \\ Department of Anesthesiology and Pain Medicine, Korean Institute of Radiological \& Medical Science, Seoul, Korea
}

Background: Congenital central hypoventilation syndrome (CCHS) is a rare disorder characterized by alveolar hypoventilation and autonomic dysregulation. Patients with CCHS have adequate ventilation while awake but exhibit hypoventilation while asleep. More severely affected patients exhibit hypoventilation both when awake and when asleep.

Case: Here, we report a case of successful spinal anesthesia and postoperative epidural analgesia in a patient with CCHS who underwent orthostatic surgery.

Conclusions: In patients with CCHS, anesthesia is used with the goal of minimizing respiratory depression to avoid prolonged mechanical ventilation. Regional anesthesia should be considered where appropriate. Continuous oxygen saturation and end-tidal carbon dioxide monitoring must be available.

Keywords: Autonomic dysregulation; Congenital central hypoventilation syndrome; Hypoxemia; Ondine’s curse; Spinal anesthesia.

Congenital central hypoventilation syndrome (CCHS), also known as Ondine's Curse, is a life-long multi-system disorder that manifests as autonomic nervous system dysregulation. One distinctive characteristic of CCHS is a dysregulation in the ventilation system, such as alveolar hypoventilation, while asleep [1].

Corresponding author: Sunam Lee, M.D., Ph.D.

Department of Anesthesiology and Pain Medicine, Korean Institute of Radiological \& Medical Science, 75 Nowon-ro, Nowon-gu, Seoul 01812, Korea

Tel: +82-2-970-1259, Fax: +82-2-970-2824

Email: sunamlee@naver.com

ORCID: https://orcid.org/0000-0003-1467-122X

Received: May 5, 2018.

Revised: July 23, 2018 (1st); October 29, 2018 (2nd).

Accepted: November 12, 2018.

Korean J Anesthesiol 2019 August 72(4): 375-380

https://doi.org/10.4097/kja.d.18.00118
Autonomic dysfunction can result in cardiac arrhythmia and asystole [2]. Patients with CCHS are at risk of pulmonary hypertension and cor pulmonale secondary to chronic hypoxia.

The development of techniques for treating CCHS have led to decrease in the mortality rate and have allowed anesthesiologists more frequently treat adolescent or adult CCHS patients.

Obviously, anesthesiologists are concerned with defective central control of ventilation, which may require postoperative mechanical ventilation, in such patients.

Given the low incidence of CCHS, the perioperative management of affected patients is based solely on previous case reports. Notably, most relevant case reports involve general anesthesia, and few discuss regional anesthesia.

We report here a case of spinal anesthesia and postoperative epidural analgesia in a patient with CCHS who underwent wide excision and reconstruction with tumor prosthesis for osteosarcoma of the distal femur.

(c) This is an open-access article distributed under the terms of the Creative Commons Attribution Non-Commercial License (http://creativecommons.org/ licenses/by-nc/4.0/), which permits unrestricted non-commercial use, distribution, and reproduction in any medium, provided the original work is properly cited. 


\section{Case Report}

A 17-year-old man, with a height of $168 \mathrm{~cm}$ and weight of $55 \mathrm{~kg}$, was admitted to the hospital to undergo a wide excision and reconstruction with tumor prosthesis for an osteosarcoma of the right distal femur. The patient had been diagnosed with CCHS based on clinical symptoms that emerged immediately after birth. At one month of age, the patient underwent a tracheostomy; at eight years of age, he underwent another surgery for the primary closure of this tracheostomy under general anesthesia. He was not admitted to the intensive care unit after the operation. He had no other previous history of anesthesia and surgery. The patient had strabismus, had fainted once previously, and had lived without restrictions in his daily life other than a few episodes of dizziness. He breathed spontaneously during wakefulness. He was placed on a bilevel positive airway pressure (PAP) $20 / 5 \mathrm{cmH}_{2} \mathrm{O}$, with a backup rate of 20 breaths/min during sleep only.

The patient's preoperative chest radiograph, electrocardiogram (ECG), echocardiography, and pulmonary function tests were normal. Blood tests revealed a hemoglobin level of 14.5 $\mathrm{g} / \mathrm{dl}$, hematocrit of $41 \%$, and blood glucose level of $118 \mathrm{mg} / \mathrm{dl}$. Arterial blood gas analysis (ABGA) performed the day before operation yielded $\mathrm{pH} 7.38, \mathrm{PaCO}_{2} 39 \mathrm{mmHg}$, and $\mathrm{PaO}_{2} 94$ $\mathrm{mmHg}$. Immediately after discontinuation of the ventilator on the morning of the operation, ABGA yielded $\mathrm{pH}$ 7.400, $\mathrm{PaCO}_{2}$ $46 \mathrm{mmHg}$, and $\mathrm{PaO}_{2} 56 \mathrm{mmHg}$ (Table 1). To avoid the effects of respiration, the patient entered the operating room without premedication. His ECG, non-invasive blood pressure (NIBP), and oxygen saturation were measured. We observed the patient's chest expansion and respiratory rate every 30 minutes by visual inspection. At the time of surgery, the patient's vital signs were as follows: blood pressure of $137 / 85 \mathrm{mmHg}$, heart rate of 109 beats/min, oxygen saturation of $100 \%$, and respiratory rate of 18

Table 1. Perioperative Vital Signs, Laboratory Blood Analysis Results

\begin{tabular}{lrrrrrr}
\hline \multicolumn{1}{c}{ Time } & \multicolumn{1}{c}{$\mathrm{T} 1$} & $\mathrm{~T} 2$ & $\mathrm{~T} 3$ & $\mathrm{~T} 4$ & $\mathrm{~T} 5$ & $\mathrm{~T} 6$ \\
\hline $\mathrm{BP}(\mathrm{mmHg})$ & $110 / 70$ & $137 / 85$ & $106 / 60$ & $105 / 58$ & $105 / 60$ & $110 / 60$ \\
$\mathrm{HR}$ (beats/min) & 110 & 109 & 98 & 101 & 86 & 102 \\
$\mathrm{RR}$ (breaths/min) & 18 & 18 & N/A & 18 & 18 & 18 \\
$\mathrm{PaCO}_{2}(\mathrm{mmHg})$ & 46 & N/A & N/A & 42 & N/A & 39 \\
$\mathrm{PaO}_{2}(\mathrm{mmHg})$ & 56 & N/A & N/A & 96 & N/A & 97 \\
$\mathrm{SpO}_{2}(\%)$ & 88 & 100 & 89 & 100 & 92 & 100
\end{tabular}

T1: immediately discontinuation of ventilation, T2: before administration of spinal anesthesia, T3: during the first hypoventilation, T4: after recovery from the first hypoventilation, T5: during the second hypoventilation, T6: after recovery from the second hypoventilation. $\mathrm{BP}$ : blood pressure, $\mathrm{HR}$ : heart rate, $\mathrm{RR}$ : respiratory rate, $\mathrm{PaCO}_{2}$ : partial carbon dioxide pressure, $\mathrm{PaO}_{2}$ : partial oxygen pressure, $\mathrm{SpO}_{2}$ : pulse oximetry oxygen saturation, N/A: not available. The patient breathed only room air throughout the perioperative period. breaths/min (Table 1).

After laying the patient in his right lateral decubitus position, spinal anesthesia was administered using a 25-gauge Whitacre spinal needle via a midline approach at the level of the L3-4 interspace. After confirming clear cerebrospinal fluid, $11 \mathrm{mg}$ of hyperbaric bupivacaine $(0.5 \%)$ and $0.3 \mathrm{mg}$ of epinephrine were injected intrathecally. Immediately after injection, the patient underwent lumbar epidural catheterization with a 20 -gauge catheter through an 18-gauge Tuohy needle at L4-5 with a loss of resistance technique using air to control postoperative pain. After 10 minutes in the right lateral decubitus position, the patient was repositioned to the supine position. Another peripheral line was placed in the left forearm to prepare for bleeding during surgery. In addition, a catheter was placed in the right radial artery in consideration of continuous arterial blood pressure monitoring and frequent blood collection during the perioperative period. To maintain the patient's body temperature, all fluid and blood were infused using a fluid warmer. His tympanic membrane temperature and blood glucose level were measured every hour. The patient exhibited some anxiety during the preparation for surgery, but he did not use sedatives. The medical staff continuously talked to and encouraged the patient to provide emotional support.

At the start of surgery, the sensory block reached T8 level, and the patient exhibited the following vital signs; blood pressure of $101 / 59 \mathrm{mmHg}$, heart rate of 101 beats/min, respiratory rate of 18 breaths/min, and oxygen saturation of $100 \%$. At 20 minutes after surgery when the patient was comfortable and the conversation was briefly interrupted, the oxygen saturation level suddenly decreased to $89 \%$ and the blood pressure increased to $106 / 60 \mathrm{mmHg}$ (Table 1 ). We couldn't observe his breathing pattern at that time. As the patient was woken up to stimulate breathing, his oxygen saturation level rapidly rebounded to $100 \%$ without requiring oxygen administration. ABGA performed immediately after recovery from hypoventilation revealed $\mathrm{PaCO}_{2} 42 \mathrm{mmHg}$ and $\mathrm{PaO}_{2} 96 \mathrm{mmHg}$ (Table 1). Two hours after the start of surgery, his oxygen saturation level again decreased to $92 \%$ (Table 1). His respiratory rate was 18 breaths/ min and his chest expansion seemed diminished at that time. His oxygen saturation level recovered to $100 \%$ immediately after respiratory stimulation, again without oxygen administration. ABGA yielded $\mathrm{PaCO}_{2} 39 \mathrm{mmHg}$ and $\mathrm{PaO}_{2} 97 \mathrm{mmHg}$ (Table 1). In both cases, the patient was awake and comfortable, although his eyes remained closed. The patient was engaged in continuous conversation and a close eye was kept on his chest movement, respiratory rate, and oxygen saturation.

Other than the two episodes of decreased oxygen saturation, no hemodynamic changes, bradycardia, or arrhythmia were observed during the operation. The patient's body temperature was $36.4-37.4^{\circ} \mathrm{C}$ his blood glucose level was $89-103 \mathrm{mg} / \mathrm{dl}$, and his 
blood oxygen, carbon dioxide, and bicarbonate levels remained within normal ranges.

At 30 minutes before the end of the operation, a $3 \mathrm{ml}$ test dose of $0.25 \%$ bupivacaine with $5 \mu \mathrm{g} / \mathrm{ml}$ epinephrine (1: $200,000)$ was used to exclude an intravascular or subarachnoid injection. An additional $2 \mathrm{ml}$ dose of $0.25 \%$ bupivacaine was administered after 10 minutes. Epidural patient-controlled analgesia (PCA) was initiated with $0.25 \%$ bupivacaine at a basal infusion rate of $2 \mathrm{ml} / \mathrm{h}$, with a $3 \mathrm{ml}$ bolus dose and 30-minute lockout time.

The duration of the operation was 2 hours and 25 minutes and the duration of anesthesia was 3 hours. The blood loss and urine output volumes were $2,000 \mathrm{ml}$ and $220 \mathrm{ml}$ respectively, and 3 units of packed red blood cells, $1,000 \mathrm{ml}$ of colloid solution, and 2,200 $\mathrm{ml}$ of crystalloid solution were administered.

The patient was transported to the post-anesthetic care unit (PACU). The postoperative monitors measured oxygen saturation, ECG, respiratory rate, and NIBP. His respiratory rate was measured by respiratory waveform (Intellivue patient monitor MX600, Philips Medizine System, Germany). ABGA revealed $\mathrm{PaCO}_{2} 30 \mathrm{mmHg}$ and $\mathrm{PaO}_{2} 98 \mathrm{mmHg}$. The observations were uneventful during the stay of $80 \mathrm{~min}$ in the PACU, and then the patient was moved to the general ward. His $\mathrm{SpO}_{2}$ was monitored after returning to the ward. No hypoxemia was detected for the remainder of the day. He was given a trial of bilevel PAP during sleep only, as his baseline schedule. Six hours after the operation, the patient complained of pain at a level of 7 on a numeral rating scale (NRS). This was controlled to an NRS level of 3 after a single intramuscular injection of $20 \mathrm{mg}$ of diclofenac and single epidural injection of a PCA bolus. ABGA on the morning of the first postoperative day yielded a $\mathrm{pH} 7.348, \mathrm{PaCO}_{2} 39.1 \mathrm{mmHg}$, and $\mathrm{PaO}_{2} 96.3 \mathrm{mmHg}$. At 24 hours after the operation, an NRS score of 3 was observed after administering two epidural bolus injections without any additional analgesics. At 48 hours after the operation, an NRS score of 3 was observed without any epidural PCA bolus or additional analgesics, and the epidural catheter was removed. For five days after the operation, proper pain control was available via a single intramuscular injection of diclofenac $20 \mathrm{mg}$ per day.

Although genetic testing, 72-hour Holter monitoring, and other tests considered necessary for patients with CCHS were recommended, the patient and his guardian wished to undergo evaluation at another hospital. Therefore, we requested the patient and his guardian to undergo periodic check-ups and evaluations at the other hospital. The remainder of his postoperative course was unremarkable, and he was discharged home on postoperative day 11 .

\section{Discussion}

CCHS is an autosomal dominant disease involving alleles with reduced penetrance. It is characterized by alveolar hypoventilation and autonomic nervous system dysregulation [1]. Most patients with CCHS exhibit symptoms during the neonatal period. Affected individuals experience adequate ventilation while awake, but exhibit hypoventilation (monotonous respiratory rate, diminutive tidal volume) or apnea during sleep; in severe cases, they exhibit hypoventilation even when awake [1]. Patients experience better ventilation during rapid eye movement (REM) sleep than during non-REM sleep [3]. They have absent or negligible ventilatory sensitivity to hypercarbia and absent or variable ventilatory sensitivity to hypoxemia during sleep [3]. Awake ventilatory responsiveness to hypercarbia and hypoxemia is generally absent, even when awake minute ventilation is adequate [3]. Patients with CCHS will occasionally demonstrate apneic pauses after the discontinuation of mechanical ventilation and before the initiation of spontaneous breathing. The hypoventilation exhibited by our patient on the morning of the operation is an example of this phenomenon.

Most cardiac abnormalities are related to autonomic dysfunction and can cause life-threatening bradyarrhythmias and asystole. Pulmonary hypertension and right heart failure are common complications secondary to chronic hypoxia. Each patient with CCHS must undergo a comprehensive evaluation to ensure the early diagnosis and appropriate treatment of significant cardiac abnormalities. Movahed et al. [2] argued that 24hour Holter monitoring should be performed every 12 months. The early recognition of significant bradyarrhythmias should prompt the clinician to consider the insertion of a permanent cardiac pacemaker.

The medical issues associated with CCHS include Hirschsprung's disease, neuroblastoma, reduced basal body temperature, episodes of profuse sweating with cool extremities, decreased pupillary light reflex, orthostatic hypotension, decrease in perception of pain, altered perception of anxiety, hypoglycemia, esophageal dysmotility, and constipation $[1,2]$.

Most patients show symptoms shortly after birth. However, in late onset CCHS (LOCCHS), either the symptoms appear more than one month after birth, or unexplained hypoventilation appears after mild sedation or anesthesia in adolescents or adults [4]. Anesthesiologists should be aware of undiagnosed LOCCHS and include this condition in the differential diagnosis of patients with unexplained postoperative respiratory depression.

In 2003, the paired-like homeobox 2B (PHOX2B) gene was identified as a CCHS-defining gene [5]. According to many reports, the $\mathrm{PHOX} 2 \mathrm{~B}$ genotype and the CCHS phenotype are closely linked; a longer polyalanine repeat expansion correlates 
with a longer duration of ventilator requirement during the day and more severe accompanying autonomic nervous system dysfunction $[1,5]$.

Gronli et al. [6] reported that the PHOX2B genotypes correlate closely with the severity of autonomic dysregulation in the heart and determines the risk of sudden death. Data from that study indicated that patients with the $P H O X 2 B$ genotypes of 20/26 and 20/27 face an increased risk of prolonged asystole. Clinically, an evaluation of $\mathrm{PHOX} 2 \mathrm{~B}$ expansion mutation could be used to predict CCHS. A better understanding of the nature of this mutation may lead to improved treatment options for patients with CCHS.

CCHS treatment aims to maintain adequate oxygenation and ventilation while the patient is awake and asleep to reduce the risk of cor pulmonale and neurological damage due to chronic hypoxia. Achievement of hyperoxia in CCHS has been shown to decrease minute ventilation during sleep. Hyperoxia may not be obtained with low-flow oxygen therapy, which is only designed to correct hypoxia. It must be stressed, however, that low-flow oxygen administration has not been used in the majority of patient with CCHS [7]. Respiratory treatment includes positive ventilators via tracheostomy, bilevel positive airway pressure, negative pressure ventilators, and diaphragmatic pacing [1].

Pre-operative evaluation of patients with CCHS begins with a medical history and physical examination to find comorbid diseases. As patients with untreated CCHS have a high risk of cardiac arrhythmia, pulmonary hypertension, and right heart failure, tests such as electrocardiography, echocardiography, and 72-hour Holter monitoring are required depending on the cardiovascular risk factors and extent of surgery. Chronic hypoxia may cause erythrocytosis; thus, it is necessary to check hemoglobin and hematocrit counts. In addition, the type of ventilator currently used, degree of respiratory support required during the day, presence of tracheostomy, history of general anesthesia, and presence of an intensive care unit after anesthesia should be confirmed [8].

Sedation may reduce the respiratory rate and should be avoided before operation [8]. Additionally, given the high prevalence of gastroesophageal reflux, preoperative prophylaxis with drugs that reduce gastric acid production and increase gastroesophageal motility may be indicated [9].

Intraoperative monitoring should be guided by the anticipated surgical procedure and the patient's underlying cardiovascular status. Patients with pulmonary hypertension and cor pulmonale associated with chronic hypoxia or with documented autonomic dysfunction may require invasive hemodynamic monitoring and central venous access. Continuous oxygen saturation and end-tidal carbon dioxide monitoring must be available, particularly in the post-anesthetic care unit. The patient may exhibit impaired temperature regulation and glucose homeostasis [9].

Although the number of reported CCHS cases is insufficient to determine precise guidelines for optimal management during the perioperative period, the use of long-acting drugs that would further depress respiration after surgery should be avoided; rather, the use of short-acting anesthetic agents such as remifentanil, propofol, sevoflurane, and desflurane is recommended [8].

Prottengeier et al. [10] selected remifentanil, which has a reliably very short context-sensitive half-time, as an analgesic and etomidate, which has a minimal hemodynamic effect, as the hypnotic. The existing literature provides conflicting recommendations regarding the maintenance of anesthesia in patients with CCHS. Some authors disapprove of volatile anesthetics, as clearance of volatile anesthetics would depend on adequate postoperative lung ventilation, which may be deficient with patients suffering from CCHS [11]. Others contend that the pharmacokinetic properties of desflurane or sevoflurane, with rapid and reliable washout after discontinuation, make them a favorable choice of anesthetic [10].

The existing literature regarding the effects of muscle relaxants on postoperative apnea is insufficient. However, a previous case report described the use of muscle relaxants in patients with CCHS and postoperative complications [4]. Therefore, proper care should be taken when using muscle relaxants. When administering muscle relaxants during the operation, neuromuscular blockade monitors should be used to ensure that neuromuscular blockade is fully reversed at the end of surgery. Sugammadex might be used to fully reverse these agents [8].

Extubation should be delayed until the patient is completely awake and after a sufficient trial of spontaneous ventilation. Conceivably, the CCHS patients will require an intensive care unit stay and mechanical ventilation for 3-4 days after abdominal and thoracic surgery $[9,12]$.

Patients with CCHS are at risk of arrhythmias, such as bradycardia and asystole, and therefore should avoid the use of drugs that aggravate these conditions. Sochala et al. [13] reported that propofol was not a good option because cases of complete atrioventricular block have been reported after propofol induction in patients with CCHS who underwent strabismus surgery.

Depending on the surgical site, regional anesthesia may be a good choice for avoiding the central effects of anesthetic agents [8]. In a previously reported case, a local anesthetic nerve catheter was used as the sole means of anesthesia [14].

Although the patient remains able to exert voluntary breathing efforts during wakefulness, waking ventilation at rest may be a concern because inactivity is frequently accompanied by hypoventilation [15]. In our case, the patient experienced two episodes of decreased oxygen saturation due to hypoventilation. In the first episode, we thought the patient was asleep, because his eyes were closed. However, during the second episode of 
hypoxemia, the patient reported that he had been awake at both times. Therefore, it is reasonable to assume that in this case, hypoventilation was a result of inactivity rather than sleep.

In patient with CCHS, the ventilatory response to hypoxia and hypercapnia are diminished. Therefore, an awake and resting patient will be at risk of hypoxia unless continuously encouraged to breathe voluntarily. Caretakers must urge the patient to breathe voluntarily, as this function remains intact.

In our patient, we underestimated the possibility of hypoventilation, because the patient lacked a history of problems with waking ventilation and because we administered regional rather than general anesthesia. Therefore, we monitored the patient's breathing pattern based only on oxygen saturation levels and visual inspection of chest expansion and respiratory rate. End-tidal $\mathrm{CO}_{2}$ monitoring would have allowed the earlier detection of hypoventilation. Pulse oximeter and end-tidal carbon dioxide monitor often provide objective evidence of early deterioration of ventilation.

Case reports have demonstrated that opioids, when administered via intravenous, intrathecal, and epidural routes, can induce significant respiratory depression in patients with CCHS [12]. Therefore, regimens using opioid-sparing analgesics such as local anesthetic infusions and non-steroidal anti-inflammatory drugs (NSAIDs) should be utilized in these patients [8]. In our case, the epidural administration of a local anesthetic and intermittent intramuscular administration of NSAIDs yielded effective non-opioid postoperative pain control.
In conclusion, for patients with CCHS, the anesthesiologist should minimize the use of anesthetic techniques and agents that might further depress postoperative respiration. Regional anesthesia is probably the method of choice. Continuous monitoring of oxygen saturation and end-tidal carbon dioxide must be available. For postoperative analgesia, a regional technique using local anesthetic agents is preferred over one using narcotics.

\section{Conflicts of Interest}

No potential conflict of interest relevant to this article was reported.

\section{Author Contributions}

\author{
Yongjoon Choi (Writing - original draft) \\ Sunam Lee (Writing - review \& editing) \\ Jiyeon Lee (Supervision) \\ Seongwon Woo (Resources)
}

\section{ORCID}

Yongjoon Choi, https://orcid.org/0000-0002-2926-3491

Sunam Lee, https://orcid.org/0000-0003-1467-122X

Jiyeon Lee, https://orcid.org/0000-0002-4871-8412

Seongwon Woo, https://orcid.org/0000-0001-7449-4742

\section{References}

1. Weese-Mayer DE, Berry-Kravis EM, Ceccherini I, Keens TG, Loghmanee DA, Trang H. An official ATS clinical policy statement: Congenital central hypoventilation syndrome: genetic basis, diagnosis, and management. Am J Respir Crit Care Med 2010; 181: 626-44.

2. Movahed MR, Jalili M, Kiciman N. Cardiovascular abnormalities and arrhythmias in patients with Ondine's curse (congenital central hypoventilation) syndrome. Pacing Clin Electrophysiol 2005; 28: 1226-30.

3. Weese-Mayer DE, Silvestri JM, Menzies LJ, Morrow-Kenny AS, Hunt CE, Hauptman SA. Congenital central hypoventilation syndrome: diagnosis, management, and long-term outcome in thirty-two children. J Pediatr 1992; 120: 381-7.

4. Mahfouz AK, Rashid M, Khan MS, Reddy P. Late onset congenital central hypoventilation syndrome after exposure to general anesthesia. Can J Anaesth 2011; 58: 1105-9.

5. Weese-Mayer DE, Berry-Kravis EM, Zhou L, Maher BS, Silvestri JM, Curran ME, et al. Idiopathic congenital central hypoventilation syndrome: analysis of genes pertinent to early autonomic nervous system embryologic development and identification of mutations in PHOX2b. Am J Med Genet A 2003; 123A: 267-78.

6. Gronli JO, Santucci BA, Leurgans SE, Berry-Kravis EM, Weese-Mayer DE. Congenital central hypoventilation syndrome: PHOX2B genotype determines risk for sudden death. Pediatr Pulmonol 2008; 43: 77-86.

7. Mather SJ. Ondine's curse and the anaesthetist. Anaesthesia 1987; 42: 394-403.

8. Basu SM, Chung FF, AbdelHakim SF, Wong J. Anesthetic considerations for patients with congenital central hypoventilation syndrome: a systematic review of the literature. Anesth Analg 2017; 124: 169-78.

9. Strauser LM, Helikson MA, Tobias JD. Anesthetic care for the child with congenital central alveolar hypoventilation syndrome (Ondine's curse). J Clin Anesth 1999; 11: 431-7.

10. Prottengeier J, Münster T, Wintermeyer P, Schmidt J. Anaesthesia for orphan disease: Haddad syndrome (Ondine-Hirschsprung disease). Eur J Anaesthesiol 2014; 31: 338-40.

11. Niazi AU, Mocon A, Varadi RG, Chan VW, Okrainec A. Ondine's curse: anesthesia for laparoscopic implantation of a diaphragm pacing 
stimulation system. Can J Anaesth 2011; 58: 1034-8.

12. Wiesel S, Fox GS. Anaesthesia for a patient with central alveolar hypoventilation syndrome (Ondine's Curse). Can J Anaesth 1990; 37: 1226.

13. Sochala C, Deenen D, Ville A, Govaerts MJ. Heart block following propofol in a child. Paediatr Anaesth 1999; 9: 349-51.

14. Visser WA, Fanyar Z, Luiten EJ. Thoracic paravertebral block for awake breast surgery in a patient with congenital central hypoventilation syndrome (Ondine's Curse). J Clin Anesth 2013; 25: 604-5.

15. Harper RM, Kumar R, Macey PM, Harper RK, Ogren JA. Impaired neural structure and function contributing to autonomic symptoms in congenital central hypoventilation syndrome. Front Neurosci 2015; 9: 415. 